\title{
Building and Optimization of the Ultra-wideband Measurement Positioning System Model
}

\author{
Yan Zhang, Lijun Mao \\ School of Intelligence Science and Information Engineering \\ Xi'an Peihua University Xi'an, China \\ 410325568@qq.com, 35909250@qq.com
}

Keywords: Ultra-wideband (UWB); Wireless sensor; Asynchronous measurement positioning

\begin{abstract}
This paper focuses on analyzing characteristics of the ultra-wideband (UWB) measurement positioning system model. To start with, problems facing the UWB measurement positioning system traditional model are briefly expounded. Concerning these problems, measures, including removing the fixed devices and equipment, weakening the boundary between measurement nodes and measured nodes as well as optimization and improvement of the measurement positioning model, are adopted to not only expand options of reference nodes during the process of distance measurement, but also maximally develop characteristics of the transceiver. To sum up, the optimized UWB measurement positioning system model put forward in this paper has promising application prospects.
\end{abstract}

\section{Introduction}

Currently, most research targeted at UWB measurement positioning technology is on the basis of synchrony. A major advantage of UWB transceiver measurement positioning lies in random deployment of sensor nodes under special conditions. Therefore, it is imperative to find out a UWB asynchronous positioning system.

However, in the real world, the advantage of the UWB transceiver mentioned above has not yet been fully reflected. It is just used for sending or receiving. Convenience and low cost of UWB design and realization call for complete utilizations. During the design process of the positioning measurement model, the advantage of the UWB transceiver should be further improved and utilized. Therefore, it is necessary to explore a new positioning system model which can achieve precise positioning and maximal utilization of the transceiver's advantage without pavement of optical fibers.

\section{Traditional UWB Measurement Positioning Model}

The traditional UWB measurement positioning algorithm adopts fiber optics to connect three or four fixed measurement points. According to the length of the optical fiber between measurement nodes, the delay time of signals is calculated so as to maintain synchronization of time. Fig. 1 below shows the UWB synchronous positioning model.

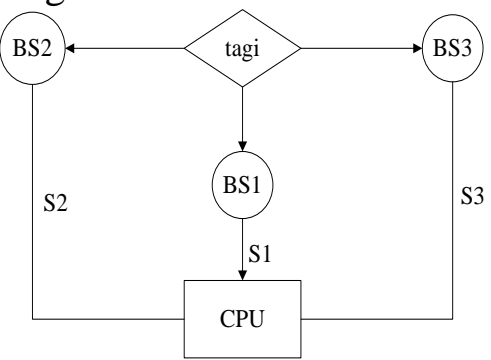

Fig. 1 UWB synchronous positioning model

CPU stands for the central processing unit, which is responsible for processing and storage of measurement of measurement data. BS1, BS2 and BS3 are three reference nodes, or the 
measurement nodes already known. The target node is denoted by tagi, which refers to the node requiring measurement. S1, S2 and S3 are the optical fibers connecting the measurement node the CPU. The arrow of the node to be measured pointing at three reference nodes represents the direction of pulse communication. In other words, as long as the pulse UWB signals sent by tagi are received by $\mathrm{BS} 1, \mathrm{BS} 2$ or BS3 during the process of communication, and when CPU sends out synchronous information and calculates the delay time of reference points, the position information of tagi can be obtained.

In the UWB synchronous positioning model demonstrated by Fig. 1, the link between tagi and BS1, BS2, BS3 or CPU cannot be realized without the help of optical fiber. In calculating the distance, CPU ensures synchronicity of the clock. All the reference nodes can have their loss calculated based on the length of the link fiber so as to acquire the most accurate time delay and measurement distance. Theoretically, the optical fiber is cheaper and more reliable. The method can ensure accuracy of positioning at a low cost. However, in the real world, it is time-consuming to pave the optical fiber, which is even inconvenient and unfeasible especially during positioning of WSNs under complex environments. Therefore, limitations of the optical fiber are obvious. Meanwhile, the reference nodes and the measured nodes mentioned above are capable of sending or receiving. On the contrary, due to easiness to realize the UWB transceiver, the two functions - receiving and sending - have been basically integrated. When the UWB transceiver is used to measure its positioning, its advantage is not displayed. In order to fully capitalize on simple design, easy realization and low cost of the UWB transceiver, research efforts should be made to explore a new positioning system model, which can achieve precise positioning and maximally utilize the advantage of the transceiver without paying the optical fiber. Currently, the positioning model has become a general research interest.

\section{Synchronous Measurement Positioning Model}

Different from the traditional positioning model, the UWB asynchronous measurement positioning system can link reference nodes and nodes to be measured without the optical fiber. However, new problems have emerged. How should the issue of clock synchronicity be resolved? How should the CPU be used to control the clock of nodes? How should the time delay be calculated? The synchronous measurement model proposed in this paper represents an attempt to solve the above research questions. The UWB asynchronous measurement positioning model is represented in Fig. 2.

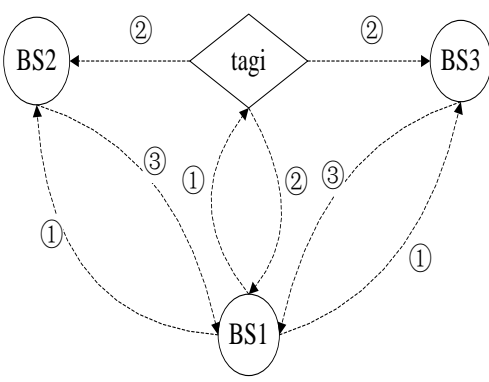

Fig. 2 UWB asynchronous measurement positioning model

As one notices in Fig. 2, the sign, (1), marked between BS1 and tagi, BS2 and BS3, respectively denotes the measurement frame sent by BS1; the sign from the measured node, tagi, to reference nodes-BS1, BS2 and BS3, respectively, stands for the response given by the measured node; the sign from the reference nodes, $\mathrm{BS} 2$ and $\mathrm{BS} 3$, to BS1 denotes the time delay information measured by BS2 and BS3, respectively.

The system assumes the distance among reference nodes as $\mathrm{d} 12$ and $\mathrm{d} 13$, respectively. $\mathrm{d} 12$ stands for the distance between BS1 and BS2; while d13 stands for the distance between BS1 and BS2. The time delay information between the measured node, tagi, and the reference nodes, BS1, BS2 and B3, is denoted by $\delta_{1 i}, \delta_{2 i}$ and $\delta_{3 i}$, respectively. According to the information acquired, the distance 
between the reference nodes and the measured node is represented by $L_{1 i}, L_{2 i}$ and $L_{3 i}$, which can be written as Eq. (1), Eq. (2) and Eq. (3), respectively:

$$
\begin{aligned}
& L_{1 i}=C * \frac{t_{1 i}-\delta_{1 i}}{2} \\
& L_{2 i}=d_{12}-L_{1 i}+C\left(t_{2 i}-\delta_{2 i}\right) \\
& L_{3 i}=d_{13}-L_{1 i}+C\left(t_{3 i}-\delta_{3 i}\right)
\end{aligned}
$$

Here, BS1 is assumed to be the main reference node with the computing capacity. During distance measurement, BS1 sends out a frame of test information, (1), first. Then, tagi, after receiving a frame of test information, (1), sent by BS1, immediately responds by sending a frame of response information, (2). As one observes in Fig. 2, under the ideal conditions, namely the four nodes are all distributed within the radius of communication, BS2, BS3 and tagi can receive the measurement frame information sent by BS1. The response frame information, (2), sent by tagi is respectively received by $\mathrm{BS} 1, \mathrm{BS} 2$ and $\mathrm{BS} 3$, respectively. When $\mathrm{BS} 2$ and $\mathrm{BS} 3$ receive the response information, (2), sent by tagi, the two reference nodes will immediately send a frame of information, (3), to BS1 in response. This information carries the time information from tagi to BS2 and BS3. Therefore, it is feasible for BS1 to measure distance based on the collected information.

Based on Fig. 2 and Eq. (1), Eq. (2) and Eq. (3), a complete set of the UWB asynchronous measurement positioning model is proposed. Compared with the traditional synchronous measurement, the model proposed in this paper outperforms either in terms of the computing capacity and energy consumption or in terms of function development. However, there is still one question mentioned above not yet solved. The method stipulated in this article is for the purpose of studying UWB measurement positioning of WSNs. Deployment of nodes is mostly random, meaning that these nodes are not fixed nodes. Hence, the model should be improved on the original basis so as to be fully applicable to WSNs.

\section{Optimization of the Asynchronous Measurement Positioning Model}

In the previous part, advantages of the asynchronous measurement positioning system compared with the synchronous measurement positioning system are analyzed. Meanwhile, the model and the measurement algorithm are provided and verified to be feasible. However, it is still limited for it to get applied to WSNs.

The linchpin to solving the above question is to eliminate fixed equipment and devices. In this way, the difference between sensor nodes will not be that clear. Similarly, the boundary between reference nodes and nodes to be measured can be weakened. In distance measurement, options of reference nodes are no longer limited. All these should be taken into consideration before designing a UWB measurement positioning model suitable for WSNs. In order to achieve the above goals, the measurement positioning model requires further improvement. The UWB asynchronous measurement and positioning improved model is shown in Fig. 3 below.

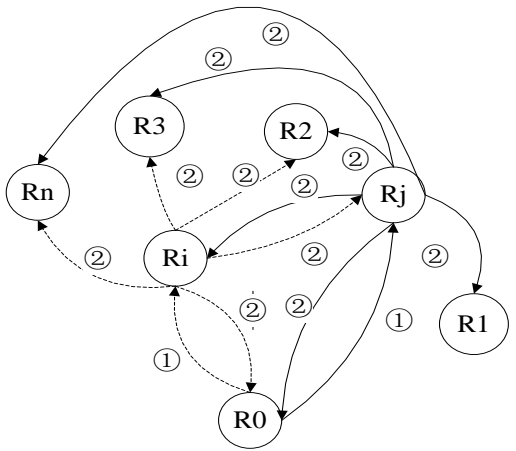

Fig. 3 Improved UWB asynchronous measurement positioning model

Fig. 3 represents the improved UWB asynchronous measurement positioning model. Assume that there have been " $n+1$ " randomly-deployed UWB WSN nodes. R0 is randomly selected as the first 
reference node. $\delta_{i j}$ denotes the time delay of UWB pulse communication from the reference node, $\mathrm{Ri}$, to the reference node, $\mathrm{Rj} ; \Delta_{i}$ denotes the time delay when the reference node, $\mathrm{Rj}$, receives the reference node, R0. $t_{i j}(\mathrm{i}=1,2,3,4,5 \ldots \ldots \mathrm{n} ; \mathrm{j}=0,1,2,3,4 \ldots \ldots \mathrm{n})$ denotes that the time difference between the time $\mathrm{Rj}$ receives the frame, (1), to the time receiving the frame, (2), after a frame of information is sent to Rj. Through corresponding calculations, Eq. (4) below can be obtained:

$t_{i j}=\sigma_{0 i}+\Delta_{i}+\sigma_{i j}-\sigma_{0 j}$

Since the time delay after the information frame, (1), is different from the one stipulated above, it is no longer an accurate value. Therefore, the time delay is represented by $\Delta_{i}$. Where, the value of $i$ ranges from 1 to $\mathrm{n}$; the value of $j$ ranges from 0 to $\mathrm{n}$. When ${ }^{j=0}$, since $\sigma_{00}=0$ and $\sigma_{0 i}=\sigma_{i 0}$. the following result can be obtained based on Eq. (5):

$t_{i 0}=2 \sigma_{0 i}+\Delta_{i}=2 \sigma_{i 0}+\Delta_{i}$

When $j=i$, since $\sigma_{i j}=0$, the following result can be obtained based on Eq. (6):

$t_{i j}=\Delta_{i}$

The distance from the main reference node, R0, to other nodes can be represented by Eq. (7) and Eq. (8), respectively:

$L_{i 0}=C \frac{t_{i 0}-\Delta_{i}}{2}$

Where, the value of $i$ ranges from 1 to $\mathrm{n}$. The distance from $\mathrm{R} 0$ to other nodes can be written as Eq. (8):

$L_{i j}=C \frac{t_{i j}-\Delta_{i}+t_{j i}-\Delta_{j}}{2}$

In the UWB asynchronous measurement positioning system mode, the functions of various nodes are the same. Every node is capable of both sending and receiving information. The measurement positioning does not require fixed reference nodes. In the positioning system, as long as the coordinate of any three or four nodes is given, the positioning coordinate of various nodes can be obtained.

\section{UWB Positioning Equation}

The quality of a measurement positioning system is decided not only by the reliability of the measurement positioning model, but also by the feasibility of the positioning algorithm. To evaluate performance of an algorithm relies on time complexity, space complexity, stability and convergence. The process of distance measurement is a process to estimate the distance for pairs of nodes in a given network. The distance from every node to the other nodes is known. Among the other nodes, $R_{i}$ can choose $\mathrm{K}$ reference nodes, $\left(R_{i} \ldots . . . R_{j}\right)$, to form a reference system. According to the following description, it clarifies its position in the reference system.

Under an ideal estimation condition without distance error, the spherical positioning technology, namely TOA (time-of-arrival) positioning, can be an effective approach. Here, the three-dimensional space, $(x, y, z)$, is observed. Distance between $R_{i}$ and the reference node, $R_{j}$, is $R_{j}\left(R_{i}\right)$, which is decided by a sphere with $D_{j i}=R_{j}\left(R_{i}\right)$ as the radius and $N_{j}$ as the center. At least fall balls are required to decide a single node in a three-dimensional space. Therefore, there should be at least four reference nodes in three-dimensional positioning. Of special note is that it is not necessary to introduce extra reference nodes to a scenario with an ideal distance. However, when the condition is not ideal, the extra reference nodes can be used to improve the system performance. This will be further analyzed in the following part. The intersection among $\mathrm{K}$ spheres can be calculated through the following equation set, namely Eq. (9): 
$\left\{\begin{array}{l}\sqrt{\left(x_{1}-x_{i}\right)^{2}+\left(y_{1}-y_{i}\right)^{2}+\left(z_{1}-z_{i}\right)^{2}} \\ \sqrt{\left(x_{2}-x_{i}\right)^{2}+\left(y_{2}-y_{i}\right)^{2}+\left(z_{2}-z_{i}\right)^{2}} \\ \cdots \cdots \\ \sqrt{\left(x_{k}-x_{i}\right)^{2}+\left(y_{k}-y_{i}\right)^{2}+\left(z_{k}-z_{i}\right)^{2}}\end{array}\right\}=\left\{\begin{array}{l}D_{1 i} \\ D_{2 i} \\ \cdots \cdots \\ D_{k i}\end{array}\right\}$

Where, $k \geq 4 . \quad x, y, z$ are the $\mathrm{x}$-coordinate, $\mathrm{y}$-coordinate and z-coordinate of the node, respectively; $\mathrm{D}$ denotes the distance between nodes. On a two-dimensional plane ${ }^{(x, y)}$ or the plane, the positioning method is similar. Combining the position of the node, ${ }^{R_{i}}$, namely $\left(x_{i}, x_{j}\right)=\operatorname{pos}\left(R_{i}\right)$, the positioning is decided by the interaction of three spheres. The intersection node can be written into the following equation:

$$
\left\{\begin{array}{l}
\sqrt{\left(x_{1}-x_{i}\right)^{2}+\left(y_{1}-y_{i}\right)^{2}} \\
\sqrt{\left(x_{2}-x_{i}\right)^{2}+\left(y_{2}-y_{i}\right)^{2}} \\
\cdots \cdots \\
\sqrt{\left(x_{k}-x_{i}\right)^{2}+\left(y_{k}-y_{i}\right)^{2}}
\end{array}\right\}=\left\{\begin{array}{l}
D_{1 i} \\
D_{2 i} \\
\cdots \ldots . . \\
D_{k i}
\end{array}\right\}
$$

Where Eq. (10), $k \geq 3, x, y$ are the $\mathrm{x}$-coordinate and $\mathrm{y}$-coordinate of the node, respecti vely. Same to Eq. (9), D denotes the distance between nodes. Fig. 4 presents the spheri cal positioning plane.

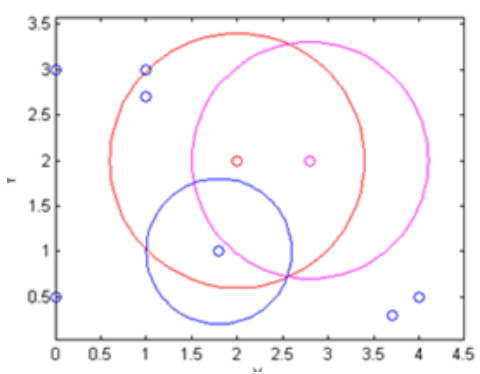

Fig. 4 Positioning plan

Spherical positioning is applicable to the condition that the common reference clock is provided for the node, $R_{i}$, and all the reference nodes. However, this is not a general condition. Lack of synchronicity, drifting or inaccuracy of the reference clock will all necessitate random experiments. The author thinks that, if there is one common reference clock provided for at least $\mathrm{K}$ reference nodes, chances are higher for precise positioning of calculations to be realized. According to the hyperbolic positioning technology, namely TDOA (time difference of arrival), the position of the reference node, $R_{i}$, is decided according to the time taken by $\mathrm{K}$ reference nodes to arrive at $R_{i}$. Here, the time of arrival of different reference nodes, after subtracted with each other, eliminates the time delay, $\delta$. According to the pair of reference nodes, $\left(R_{n}, R_{n-1}\right)$, the following Eq. (11) can be obtained:

$D_{n i}-D_{(n-1) i}=c\left(\tau_{n i}+\delta\right)-c\left(\tau_{(n-1) i}+\delta\right)=c\left(\tau_{n i}-\tau_{(n-1) i}\right)$

In a three-dimensional space, the position of the node, $R_{i} \sqrt{b^{2}-4 a c}$, is decided by the intersection of hyperboloids in the space, which can be written as Eq. (12):

$$
\left\{\begin{array}{l}
\sqrt{\left(x_{2}-x_{i}\right)^{2}+\left(y_{2}-y_{i}\right)^{2}+\left(z_{2}-z_{i}\right)^{2}}-\sqrt{\left(x_{1}-x_{i}\right)^{2}+\left(y_{1}-y_{i}\right)^{2}+\left(z_{1}-z_{i}\right)^{2}} \\
\sqrt{\left(x_{3}-x_{i}\right)^{2}+\left(y_{3}-y_{i}\right)^{2}+\left(z_{3}-z_{i}\right)^{2}}-\sqrt{\left(x_{2}-x_{i}\right)^{2}+\left(y_{2}-y_{i}\right)^{2}+\left(z_{2}-z_{i}\right)^{2}} \\
\cdots \cdots \\
\sqrt{\left(x_{k}-x_{i}\right)^{2}+\left(y_{k}-y_{i}\right)^{2}+\left(z_{k}-z_{i}\right)^{2}}-\sqrt{\left(x_{k-1}-x_{i}\right)^{2}+\left(y_{k-1}-y_{i}\right)^{2}+\left(z_{k-1}-z_{i}\right)^{2}}
\end{array}\right\}=\left\{\begin{array}{l}
D_{2 i}-D_{1 i} \\
D_{3 i}-D_{2 i} \\
\cdots \ldots \\
D_{k i}-D_{(k-1) i}
\end{array}\right\}
$$

In Eq. (12), $k \geq 4$ should be satiated; $x, y$ are the $\mathrm{x}$-coordinate and the y-coordinate of the node; $\mathrm{D}$ denotes the distance between nodes.

In a two-dimensional space, however, Eq. (13) below can be used for computing: 


$$
\left\{\begin{array}{l}
\sqrt{\left(x_{2}-x_{i}\right)^{2}+\left(y_{2}-y_{i}\right)^{2}}-\sqrt{\left(x_{1}-x_{i}\right)^{2}+\left(y_{1}-y_{i}\right)^{2}} \\
\left(x_{3}-x_{i}\right)^{2}+\left(y_{3}-y_{i}\right)^{2}-\sqrt{\left(x_{2}-x_{i}\right)^{2}+\left(y_{2}-y_{i}\right)^{2}} \\
\cdots \cdots \\
\sqrt{\left(x_{k}-x_{i}\right)^{2}+\left(y_{k}-y_{i}\right)^{2}}-\sqrt{\left(x_{k-1}-x_{i}\right)^{2}+\left(y_{k-1}-y_{i}\right)^{2}}
\end{array}\right\}=\left\{\begin{array}{l}
D_{2 i}-D_{1 i} \\
D_{3 i}-D_{2 i} \\
\cdots \ldots \\
D_{k i}-D_{(k-1) i}
\end{array}\right\}
$$

Eq. (13) requires $k \geq 3 . x, y$ are the $\mathrm{x}$-coordinate and the $\mathrm{y}$-coordinate of the node; $\mathrm{D}$ denotes the distance between nodes.

Fig. 5 provides positioning calculation using hyperbolas in the two-dimensional space.

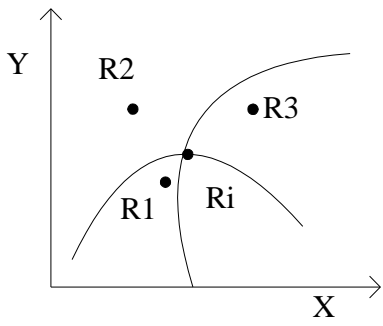

Fig. 5 Positioning in the two-dimensional space using hyperbolas

In Fig. 5, R1, R2, R3 and Ri are reference nodes in the two-dimensional space. The hyperbolic positioning requires a precise clock shared by all reference nodes. However, this does not mean there should be strict synchronicity between the reference node and the target node. Compared with the network based on infrastructure, the coordination between equipment nodes is easier to realize and maintain. Therefore, it can be widely used for positioning of the cellular networks. As a reference node, the base station allows a mobile node to acquire its own position.

Both spherical positioning and hyperbolic positioning call for information without distance measurement error. Nevertheless, thermal noise can easily cause positioning error during the distance measurement process. As a result, nodes fail to achieve ideal distance estimation among them. Under the condition, neither spherical positioning nor hyperbolic positioning is feasible. In other words, Eq. (9) and Eq. (12) are both not substantiated. Eq. (6) fully illustrates the problem.

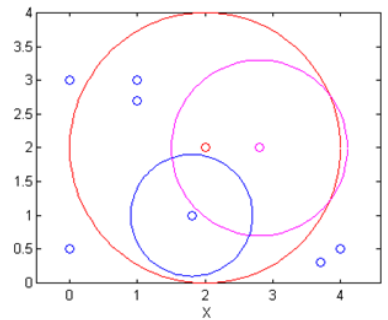

Fig. 6 Influence of distance measurement error on spherical positioning

Fig. 6 shows the number and communication radius of various nodes. Due to influence of the distance measurement error on spherical positioning of nodes in a two-dimensional space, the reference node outside the communication radius might be included in the radius and used for calculation again. The cumulative error and redundancy brought by cumulative circulation will lead to a continuous increase in error. Therefore, the linchpin to solving this problem is to solve the positioning equation.

\section{Conclusions}

To sum up, this paper analyzes the existing UWB synchronous measurement positioning model. Considering specialness of WSNs and great limitation in the current synchronous model concerning its time synchrony, a UWB asynchronous measurement positioning technology is proposed for WSNs. Problems existing in the current UWB asynchronous positioning algorithm to be solved are first examined. Meanwhile, in order to maximize development of advantages of UWB, the original model is further improved to make it more applicable to positioning of WSNs. 


\section{Acknowledgment}

This work was supported by the Special Scientific Research Project of Shaanxi Provincial Ed ucation Department (No.17JK1059) and Shaanxi Province Education Science Planning Topic( No.SGH17H461).

\section{References}

[1] Ibriq J,Mahgoub I Cluste-Based routing in wireless sensor networks: Issues and Challenges .Proc.of the 2004 Int'l SymP. On Performance Evaluation of Computer Telecommunication Systems,SanJose,2004:709-766.

[2] Tang Yong,Zhou Ming-Tian,Zhang Xin. Overview of Routing Protocols in Wireles Sensor NetworkS[J].JoumalofSoftware,2006:410-421.

[3] HaasZ,HalpernJ, LiL.Gossip-Based adhoc routing. IEEE/ACMTransaetionson Networking, Piseataway: IEEE Press,2006:479-491.

[4] HeinzelmanW,ChandrakasanA, BalakrishnanH.An application-specific protocol architecture for wireless microsen-sornetworks Wireless Communications[J]. IEEE Transactions,2002, 1(4): 660-670.

[5] Chan H, Perrig A. ACE: An emergent algorithm for highly uniform cluster formation. In: Proc. of the 1st European Workshop on Wireless Sensor Networks. LNCS 2920, Berlin: Springer-Verlag, 2004. 154-171.

[6] Manjeshwar A, Grawal DP. TEEN: A protocol for enhanced efficiency in wireless sensor networks. In: Proc. of the 15th Parallel and Distributed Processing Symp. San Francisco: IEEE Computer Society, 2001. 2009-2015. 